\title{
THE SECOND CONJUGATE ALGEBRAS OF BANACH ALGEBRAS
}

\author{
PAK-KEN WONG
}

Department of Mathematics

Seton Hall University

South Orange, NJ 07079

(Received December 12, 1990)

ABSTRACT. In this paper, we study Arens regularity of a Banach algebra $A$. In particular, we give characterizations for $A$ to be Arens regular.

KEY WORDS AND PHRASES. Banach algebra, Arens products, Arens regularity, weakly compact operators.

1991 AMS SUBJECT CLASSIFICATION CODES. Primary 46H10; Secondary 46H99.

\section{INTRODUCTION.}

Let $A$ be a Banach algebra. It is an interesting and difficult problem to determine whether $A$ is Arens regular. Many papers have been written on this subject. For example, see [2], [5], [9], [10], [11] and [12]. In particular let $A$ be a $B^{*}$-algebra. It is well known that $A$ is Arens regular. However, it is not easy to prove this result. There are many different proofs of this result. For example, see [4], [5], and [8].

In this paper, we give characterizations for $A$ to be Arens regular. It follows from this result and a result of C.A. Akemann that a $B^{*}$-algebra is Arens regular. We also show that if $A$ is a Banach algebra which is Arens regular, then any closed subalgebra of $A$ is also Arens regular.

2. NOTATION AND PRELIMINARIES.

Definitions not explicitly given are taken from Rickart [7].

Let $A$ be a Banach algebra and let $A^{*}$ and $A^{* *}$ be the conjugate and second conjugate spaces of $A$. We will denote by $\pi$ the canonical embedding of $A$ into $A^{* *}$. The two Arens products on $A^{* *}$ are defined in stages according to the following rules (see [3]). Let $x, y \in A, f \in A^{*}$ and $F, G \in A^{* *}$.

Define $f o x$ by $(f o x)(y)=f(x y)$. Then $f o x \in A^{*}$.

Define $G$ of by $(G o f)(x)=G(f o x)$. Then $G o f \in A^{*}$.

Define $F o G$ by $(F o G)(f)=F(G o f)$. Then $F o G \in A^{* *}$.

$A^{* *}$ is a Banach algebra under the Arens product $o$, and we denote this algebra by $\left(A^{* *}, o\right)$.

Define $x o^{\prime} f$ by $\left(x o^{\prime} f\right)(y)=f(y x)$. Then $x o^{\prime} f \in A^{*}$.

Define $f o^{\prime} F$ by $\left(f o^{\prime} F\right)(x)=F\left(x o^{\prime} f\right)$. Then $f o^{\prime} F \in A^{*}$.

Define $F o^{\prime} G$ by $\left(F o^{\prime} G\right)(f)=G\left(f o^{\prime} F\right)$. Then $F o^{\prime} G \in A^{* *}$.

$A^{* *}$ is a Banach algebra under the Arens product $o^{\prime}$ and we denote this algebra by $\left(A^{* *}, o^{\prime}\right)$.

Both of the Arens products extend the given multiplication on $A$ when $A$ is canonically embedded in $A^{* *}$. In general, $o$ and $o^{\prime}$ are distinct on $A^{* *}$. If they agree on $A^{* *}$, then $A$ is called Arens regular.

In this paper, all algebras and linear spaces under consideration are over the complex field $C$. 
3. ARENS REGULARITY FOR BANACH ALGEBRAS.

Let $A$ be a Banach algebra and $f \in A^{*}$. Define $L_{f}: A \rightarrow A^{*}$ by

$$
L_{f}(x)=f o x \quad(x \in A) .
$$

Then $L_{f}$ is clearly a continuous linear operator from $A$ to $A^{*}$.

For each $F \in A^{* *}$, define $F . L_{f}$ by

$$
F . L_{f}(x)=F\left(L_{f}(x)\right)=F(f o x)=(F o f)(x) .
$$

Then $F . L_{f} \in A^{*}$. Define $L_{f}^{*}: A^{* *} \rightarrow A^{*}$ by

$$
L_{f}^{*}(F)=F \cdot L_{f}=F o f \quad\left(F \in A^{* *}\right) .
$$

Then $L_{f}^{*}$ is clearly a continuous linear operator from $A^{* *}$ to $A^{*}$.

For each $F \in A^{* *}$, define $F . L_{f}^{*}$ by

$$
F . L_{f}^{*}(G)=F\left(L_{f}^{*}(G)\right)=F(G o f) \quad\left(G \in A^{* *}\right) .
$$

Then $F . L_{f}^{*} \in A^{* * *}$. Finally, we define $L_{f}^{* *}: A^{* *} \rightarrow A^{* * *}$ by

$$
L_{f}^{* *}(F)=F . L_{f}^{*} \quad\left(F \in A^{* *}\right)
$$

Then clearly $L_{f}^{* *}$ is a continuous linear operator from $A^{* *}$ to $A^{* * *}$.

THEOREM 1. Let $A$ be a Banach algebra. Then the following statements are equivalent:

(1) $A$ is Arens regular.

(2) For each $f \in A^{*}, L_{f}^{* *}\left(A^{* *}\right)$ is contained in $\pi\left(A^{*}\right)$, where $\pi\left(A^{*}\right)$ is a subspace of $A^{* * *}$.

(3) For each $f \in A^{*}, L_{f}$ is weakly compact.

(4) Let $F \in A^{* *}$ and $\left\{x_{\alpha}\right\}$ a bounded net in $A$. If $\pi\left(x_{\alpha}\right) \rightarrow F$ weakly, then $f o^{\prime} F$ is a weakly limit point of $\left\{f o x_{\alpha}\right\}$.

PROOF. (1) $\Rightarrow(2)$. Assume (1). Let $F, G \in A^{* *}$. Then $L_{f}^{* *}(f)=F \cdot L_{f}^{*}$ and by (1)

$$
F . L_{f}^{*}(G)=F\left(L_{f}^{*}(G)\right)=F(G o f)=(F o G)(f)=\left(F o^{\prime} G\right)(f)=G\left(f o^{\prime} F\right)=\pi\left(f o^{\prime} F\right)(G) .
$$

Therefore $F . L_{f}^{*}=\pi\left(f o^{\prime} F\right) \in \pi\left(A^{*}\right)$ and so $L_{f}^{* *}(F)=F . L_{f}^{*} \in \pi\left(A^{*}\right)$. This proves (2).

(2) $\Rightarrow(3)$. This follows immediately from $[6 ;$ p. 482, Theorem 2].

(3) $\Rightarrow$ (4). Assume that $L_{f}$ is weakly compact. Let $F$ and $G \in A^{* *}$. Then by Goldstine's theorem [6; p. 424, Theorem 5] there exists a bounded net $\left\{x_{\alpha}\right\}$ in $A$ such that $\pi\left(x_{\alpha}^{\prime}\right) \rightarrow F$ weakly. Similarly, there exists a bounded net $\left\{y_{\beta}\right\}$ such that $\pi\left(y_{\beta}\right) \rightarrow G$ weakly. Since $L_{f}$ is weakly compact, we can assume that $L_{f}\left(x_{\alpha}\right) \rightarrow g$ weakly for some $g \in A^{*}$. Hence $f o x_{\alpha} \rightarrow g$ weakly. Therefore

$$
\begin{aligned}
\lim _{\alpha} G\left(f o x_{\alpha}\right) & =G(g)=\lim _{\beta} \pi\left(y_{\beta}\right)(g)=\lim _{\beta} \lim _{\alpha} \pi\left(y_{\beta}\right)\left(f o x_{\alpha}\right) \\
& =\lim _{\beta} \lim _{\alpha} f\left(x_{\alpha} y_{\beta}\right)=\lim _{\beta} \lim _{\alpha}\left(y_{\beta} o^{\prime} f\right)\left(x_{\alpha}\right) \\
& =\lim _{\beta} \lim _{\alpha} \pi\left(x_{\alpha}\right)\left(y_{\beta^{\prime}} f\right)=\lim _{\beta} F\left(y_{\beta^{\prime}} f\right) \\
& =\lim _{\beta}\left(f o^{\prime} F\right)\left(y_{\beta}\right)=\lim _{\beta} \pi\left(y_{\beta}\right)\left(f o^{\prime} F\right)=G\left(f o^{\prime} F\right) .
\end{aligned}
$$

Therefore $f o^{\prime} F$ is a weak limit point of $\left\{f_{0 x}\right\}$. This proves (4).

(4) $\Rightarrow$ (1). Assume (4). Let $F, G \in A^{* *}$. Then by Goldstine's theorem, there exists a bounded net $\left\{x_{\alpha}\right\}$ in $A$ such that $\pi\left(x_{\alpha}\right) \rightarrow F$ weakly. Since $f o^{\prime} F$ is a weakly limit point of $\left\{f o x_{\alpha}\right\}$, we can assume that

$$
G\left(f o^{\prime} F\right)=\lim _{\alpha} G\left(f o x_{\alpha}\right)=\lim _{\alpha}(G o f)\left(x_{\alpha}\right)=\lim _{\alpha} \pi\left(x_{\alpha}\right)(G o f)=F(G o f)=F o G(f)
$$


Therefore $\left(F o^{\prime} G\right)(f)=G\left(f o^{\prime} F\right)=F o G(f)$ and so $A$ is Arens regular. This completes the proof of the theorem.

COROLLARY 2. Let $A$ be a Banach algebra such that each continuous linear map $T$ of $A$ into $A^{*}$ is weakly compact, then $A$ is Arens regular.

PROOF. Since each $L_{f}\left(f \in A^{*}\right)$ is weakly compact, $A$ is Arens regular by Theorem 1 .

Let $A$ be a $B^{*}$-algebra and $B$ a Banach space such that $B^{*}$ is a $W^{*}$-algebra. Then by $[1 ;$ p.293, Corollary II.9], any continuous linear map $T$ of $A$ into $B$ is weakly compact. Therefore it follows from Corollary 1 that $A$ is Arens regular. The property that "any continuous linear map $T$ of $A$ into $B$ is weakly compact" is a very strong one. In order for $A$ to be Arens regular, we need only to show that $L_{f}$ is weakly compact for all $f$ in $A^{*}$. Therefore, a simple proof for a $B^{*}$-algebra to be Arens regular may exist.

\section{SUBALGEBRAS OF A BANACH ALGEBRA WHICH IS ARENS REGULAR.}

Let $A$ be a Banach algebra which is Arens regular. It is well known that a subalgebra of $A$ may not be Arens regular. In fact, let $M$ be the group algebra of an infinite abelian locally compact group. Then $M$ is an $A^{*}$-algebra. Let $A$ be the completion of $M$ in an auxiliary norm. By [5; p.857, Theorem 3.14] $M$ is not Arens regular. Since $A$ is a $B^{*}$-algebra, $A$ is Arens regular.

Let $A$ be a Banach algebra and $M$ a closed subalgebra of $A$. For each $f \in A^{*}$, we define $f_{M}$ by $f_{M}(x)=f(x)$ for all $x \in M$. Then $f_{M} \in M^{*}$.

THEOREM 3. Let $M$ be a closed subalgebra of $A$. If $A$ is Arens regular, then so is $M$.

PROOF. Let $f \in M^{*}$. Then there exists some $\tilde{f} \in A^{*}$ such that $\tilde{f}_{M}=f$. Let $F \in M^{* *}$. Define $\tilde{F}$ by

$$
\tilde{F}(g)=F\left(g_{M}\right) \quad\left(g \in A^{*}\right) .
$$

Then it is clear that $\tilde{F} \in A^{* *}$. Since $A$ is Arens regular, by Theorem $1, L_{\tilde{f}}$ is weakly compact on $A$. Let $\left\{x_{\alpha}\right\}$ be a bounded net in $M$, then $L_{\tilde{f}}\left(x_{\alpha}\right)=\tilde{f} o x_{\alpha} \rightarrow g$ weakly for some $g \in A^{*}$. Since $\left(\tilde{f} \circ x_{\alpha}\right)_{M}=f o x_{\alpha} \in M^{*}$, it follows that

$$
F\left(g_{M}\right)=\tilde{F}(g)=\lim _{\alpha} m \tilde{F}\left(\tilde{f} o x_{\alpha}\right)=\lim _{\alpha} F\left(\left(\tilde{f} o x_{\alpha}\right)_{M}\right)=\lim _{\alpha} m F\left(f o x_{\alpha}\right) .
$$

Therefore $L_{f}\left(x_{\alpha}\right) \rightarrow g_{M}$ weakly and so by Theorem 1, M is Arens regular. This completes the proof.

\section{REFERENCES}

1. AKEMANN, C.A., The dual space of an operator algebra, Trans. Amer. Math. Soc. 126 (1967), 286-302.

2. ALEXANDER, F.E., The bidual of $\mathrm{A}^{*}$-algebras of the first kind, J.London Math. Soc. 12 (1975), 1-6.

3. ARENS, R.E., The adjoint of a bilinear operation, Proc. Amer. Soc. 2 (1951), 839-848.

4. BONSALL, F.F. \& DUNCAN, J., Complete Normed Algebras, Springer, Berlin, 1973.

5. CIVIN, P \& YOOD, B., The second conjugate space of a Banach algebra as an algebra, Pacific J. Math. 11 (1961), 847-870.

6. DUNFORD, N. \& SCHWARTZ, J., Linear operators. I: General theory, Pure and Appl. Math. 7, Interscience, New York, 1958.

7. RICKART, C.E., General theory of Banach algebras, University Series in Higher Math, Van Nostrand, Princeton, N.J., 1960.

8. TOMITA, M., The second dual of a $C^{*}$-algebra, Mem. Fac. Kyushu Univ. Ser. A. 21 (1967), 185-193. 
9. TOMIUK, B.J., Biduals of Banach algebras which are ideals in a Banach algebra, Acta Math. Hung. 52 (3-4) (1988), 255-263.

10. WONG, P.K., Modular annihilator A*-algebras, Pacific J. Math. 37 (1971), 825-834.

11. WONG, P.K., On the Arens products and certain Banach algebras, Trans. Amer. Math. Soc. $\underline{180}$ (1973), 437-448.

12. WONG, P.K., The second conjugates of certain Banach algebras, Canadian J. Math. 27 (1975), 1029-1035.

13. WONG, P.K., Arens product and the algebra of double multipliers, Proc. Amer. Math. Soc. 94 (1985), 441-444.

14. WONG, P.K., Arens product and the algebra of double multipliers II, Proc. Amer. Math. Soc. 100 (1987), 447-453. 


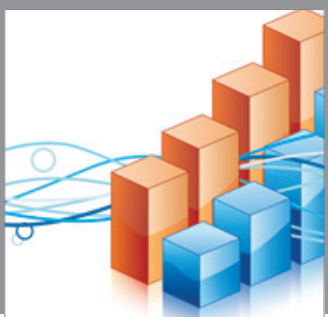

Advances in

Operations Research

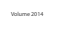

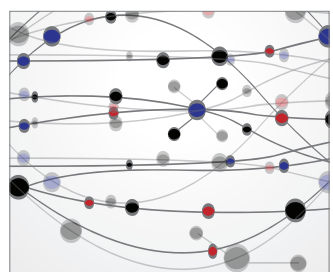

\section{The Scientific} World Journal
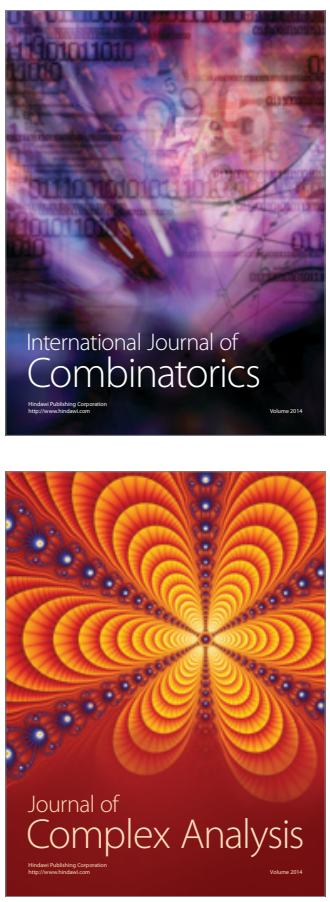

International Journal of

Mathematics and

Mathematical

Sciences
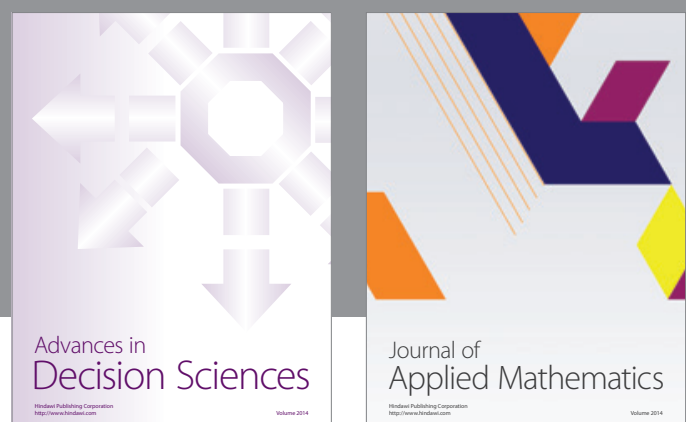

Journal of

Applied Mathematics
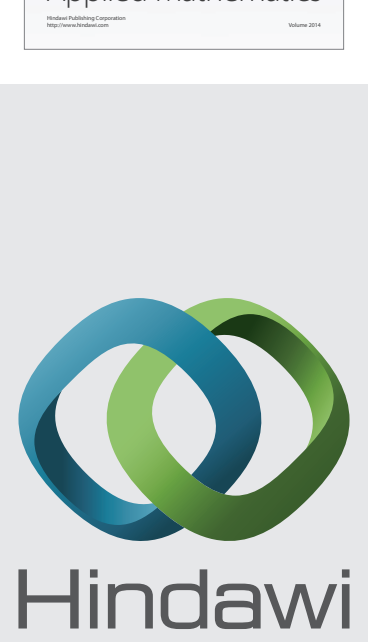

Submit your manuscripts at http://www.hindawi.com
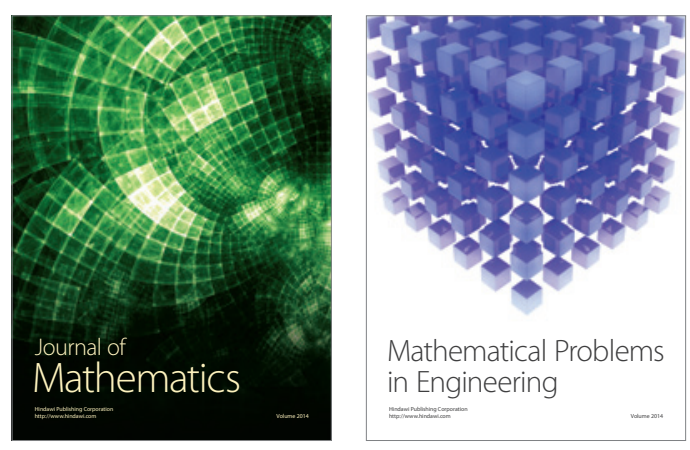

Mathematical Problems in Engineering
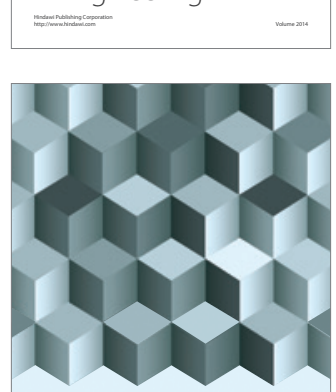

Journal of

Function Spaces
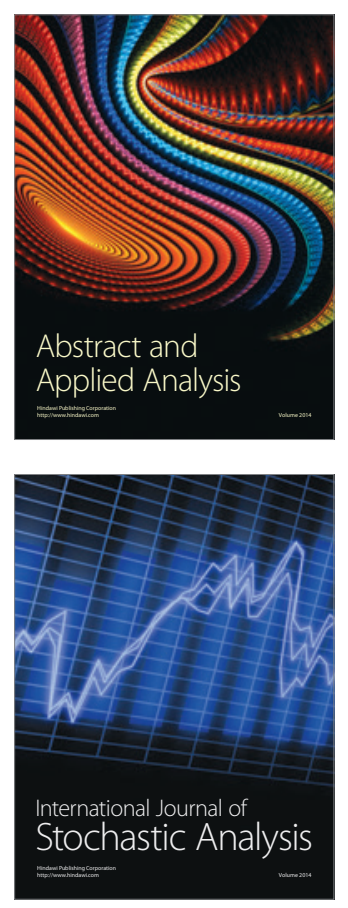

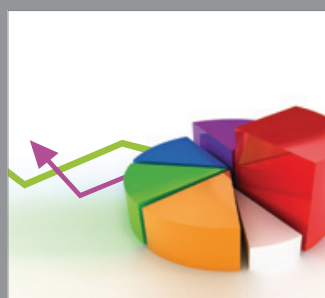

ournal of

Probability and Statistics

Promensencen
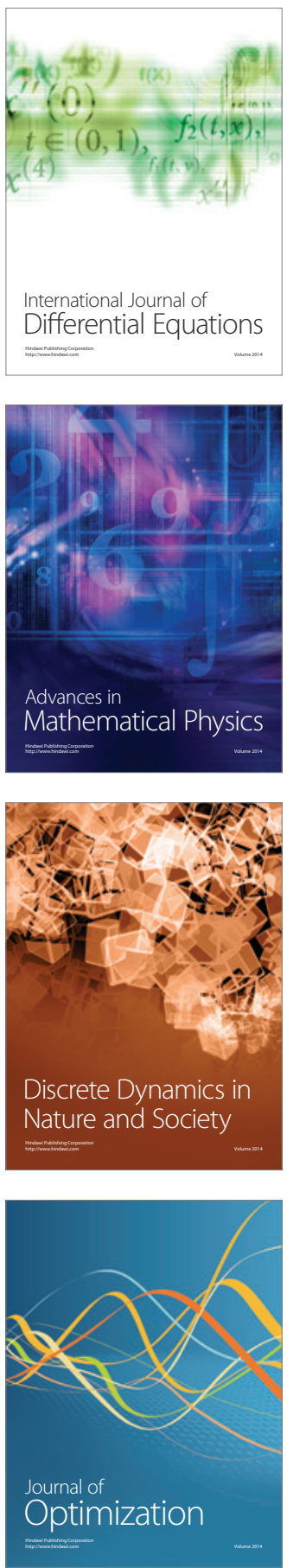